\title{
On the SN 1993J Radio Shell Structure
}

J.M. Marcaide ${ }^{1}$, I. Martí-Vidal ${ }^{1}$, E. Ros ${ }^{2}$, A. Alberdi ${ }^{3}$, J.C. Guirado ${ }^{1}$, L. Lara ${ }^{3,4}$, M.A. Pérez-Torres ${ }^{3}$, and K.W. Weiler ${ }^{5}$

${ }^{1}$ Departamento de Astronomía, Universitat de València, 46100 Burjassot, Spain; J.M. MarcaideQuv.es

2 Max-Planck-Institut für Radioastronomie, 53121 Bonn, Germany

${ }^{3}$ IAA-CSIC, Apdo. Correos 3004, 18080 Granada, Spain

${ }^{4}$ Universidad de Granada, 18071 Granada, Spain

${ }^{5}$ Naval Research Laboratory, Code 7213, Washington DC 20375-5320, USA

Summary. An accurate measurement of the expansion deceleration of SN 1993J depends on how well the shell size and its emission structure are known. With the goal of determining the emission structure of the shell, we have developed a new approach, which we call "Green Function Deconvolution" (GFD), based on iterative use of Green functions on the sky plane to reconstruct the radial emission profiles of spherically symmetric sources. This approach works reasonably well in the case of optically thin emitting sources, which is not the case for SN 1993J since, as we find, the emission from the central part of SN 1993J further away from us is strongly or totally absorbed. We describe the GFD method and present our findings about the emission structure of the shell. We also present the expansion of SN 1993J based on a method complementary to GFD, which will be described elsewhere.

\section{Introduction}

The determination of the characteristics of expansion of SN 1993J require accurate measurements of the true size of SN 1993J at each epoch. The images of SN 1993J obtained over 10 years are all circularly symmetric to a great extent and suggest an isotropic expansion. Actually, expansion decelerations have been determined $[2,3,6,10]$. However, although compatible with each other, these determinations have been obtained differently. The group led by Marcaide has made the size measurements directly on the radio images, that is on the sky plane, while the group led by Bartel has made those size measurements on the Fourier plane assuming a shell model for the source with a given shell width and optically thin emission [4]. As shown by Alberdi and Marcaide [1] (see their Fig. 4) and [4] systematic trends in the expansion measurements are present.

In principle, the determination of the size of the radio supernova SN 1993J depends on the shell model used and there are no a priori reasons to assume a particular shell model. In practice, the determination of the external radius of the radio supernova is easier than the determination of the shell model, but the former determination is contaminated by lack of determination of the latter. The best approach would be to determine both accurately and 

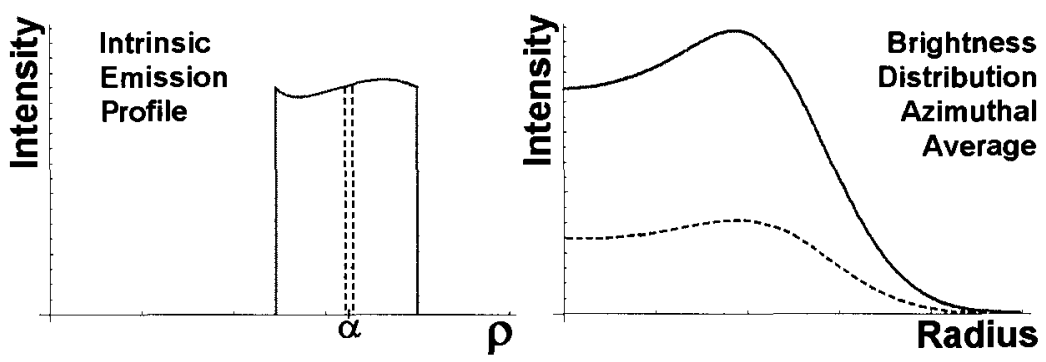

Fig. 1. (Left) A schematic of an arbitrary radial emission profile, indicating that it consists of a sum of very narrow profiles (dashed), which in turn correspond to the emission of very narrow 3D spherical shells; (Right) Schematic of the azimuthal average of the $2 \mathrm{D}$ brightness distribution generated by the emission of the 3D-shell corresponding to the radial profile given at left. It corresponds to the sum of Green Functions of very narrow profiles (dashed line, $G_{\sigma}(r, \alpha)$, see text) which add up to the given emission profile.

simultaneously. We have developed a tool for such purpose. The method, presented in this contribution, should be a good tool for cases of circular structures resulting from optically thin emission and in conditions of high signal to noise.

\section{GFD Method}

Let us consider the brightness distribution $I_{\alpha, \sigma}(r, \theta)$ due to a very (infinitesimally) thin spherical shell of radius $\alpha$ with uniform volume emissivity, once convolved by a beam of size $\sigma$. Let us indicate schematically such emission by a dotted profile on Fig. 1 (left). The azimuthally averaged emission $I_{\alpha, \sigma}(r)$ can thus be analytically expressed as:

$$
G_{\sigma}(r, \alpha)=\exp \left(\frac{-r^{2}}{2 \sigma^{2}}\right) \int_{\rho=0}^{\alpha} \rho d \rho \frac{\alpha}{\left(\alpha^{2}-\rho^{2}\right)^{\frac{1}{2}}} \exp \left(\frac{-\rho^{2}}{2 \sigma^{2}}\right) \operatorname{Bessel}\left(0, \frac{r \rho}{\sigma^{2}}\right)
$$

where $\operatorname{Bessell}(0, x)$ is the zeroth order modified Bessel Function of the First Kind. The above expression is thus a Green Function $G_{\sigma}(r, \alpha)$ which takes us from the emission profile on Fig. 1 (left) to the azimuthal average of the corresponding brightness distribution given on Fig. 1 (right).

The brightness distribution due to any optically thin shell of finite width will thus be a linear sum of the brightness distributions from a large number of very thin shells as indicated by the profile on Fig. 1 (left). In other words, as shown in Fig. 1 (right), the emission $I_{\text {shell, } \sigma}(r)$ from a finite width shell will be a weighted sum of elementary contributions $I_{\alpha, \sigma}(r)$, that is, a weighted sum 
of Green functions $G_{\sigma}(r, \alpha)$, where the weights can be chosen to reproduce any emission profile by the appropriate $S(\alpha)$ in the expression

$$
I_{\text {shell }, \sigma}(r)=\int_{\alpha_{1}}^{\alpha_{2}} S(\alpha) G_{\sigma}(r, \alpha) d \alpha
$$

Let us now consider a map of a radio source of circular symmetry (reconstructed with a beam of size $\sigma$ ) described by the brightness distribution $B_{\sigma}(x, y)$ in Cartesian coordinates, or equivalently, $B_{\sigma}(r, \Theta)$ in polar coordinates. The azimuthal average of $B_{\sigma}(r, \Theta)$ yields $B_{\sigma}(r)$, which is equivalent to $I_{\sigma}(r)$ conceptually but is degraded with respect to it by radio noise, data calibration errors, image reconstruction errors, etc, say in a VLBI observation.

The basic idea of GFD is that $B_{\sigma}(r)$ can be "deconvolved" using functions of the type $G_{\sigma}(r, \alpha)$ to obtain an emission profile of the type of $S(\alpha)$.

\section{GFD on Test Maps}

We have generated a noiseless map which corresponds to the emission of an optically thin spherical shell of finite width (30\% of outer radius) and we have made the azimuthal average of the map. The resulting intensity profile and the schematic of the shell emission profile (flat profile within the shell boundaries) is shown in Fig. 2 as the continuous line and light-shaded profile, respectively. Also in Fig. 2 the shell emission profile recovered after a GFD and the residuals are shown as a dark-shaded profile and a dashed line, respectively. As it can be seen, the GFD recovers the original emission profile rather well.

In order to further test the goodness of the GFD method we have generated the same model as in Fig. 2 but now we have added noise to the synthetic data. The added amount of noise (corresponding to random Gaussian errors of $10 \%$ in amplitude and 10 degrees in phase) simulates realistic observational conditions in VLBI. The resulting intensity profile, GF-deconvolved emission profile, and intensity residuals after GFD are shown in Fig. 3. The reconstruction of the shell profile is not as reliable as before now that noise and calibration errors are present. The level of emission appears enhanced in the inner part, the outer profile appears depleted, and the width of the shell appears much narrower. It is not a satisfactory reconstruction. The difficulties of the method have to do with the asymmetry of the Green Functions of the very narrow shells which are used as basic elements in the deconvolution. This asymmetry causes biases and some of them can be accounted for in a proper deconvolution strategy.

However, we have also found that in deconvolutions of real maps (see below in Sect. 4 and Fig. 5) we always obtain large negative residuals at the source center region which indicate that our maps contain less emission at 


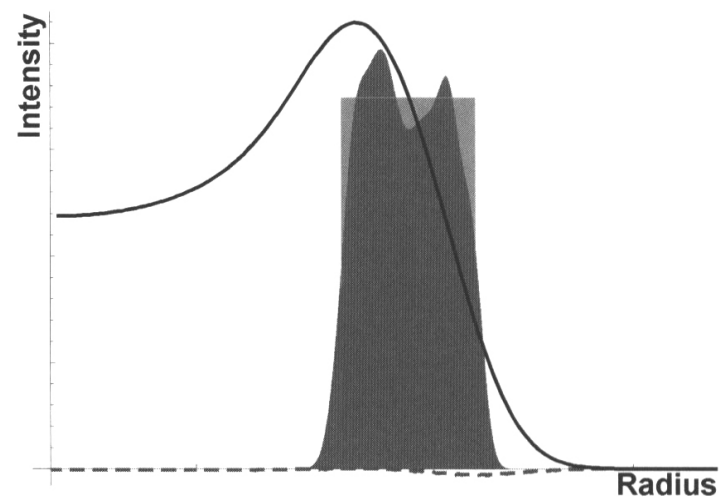

Fig. 2. Intensity profile (continuous line) corresponding to an azimuthal average of the brightness distribution due to a shell of flat radial emission profile (lightshaded) and finite width (30\% of outer radius) and reconstructed emission profile (dark-shaded) using GFD. No noise has been added to the synthetic data which produce the brightness distribution. The residuals of the GFD (dashed line) are almost zero, as expected. The beam sizes used also correspond to $30 \%$ of the outer radius.

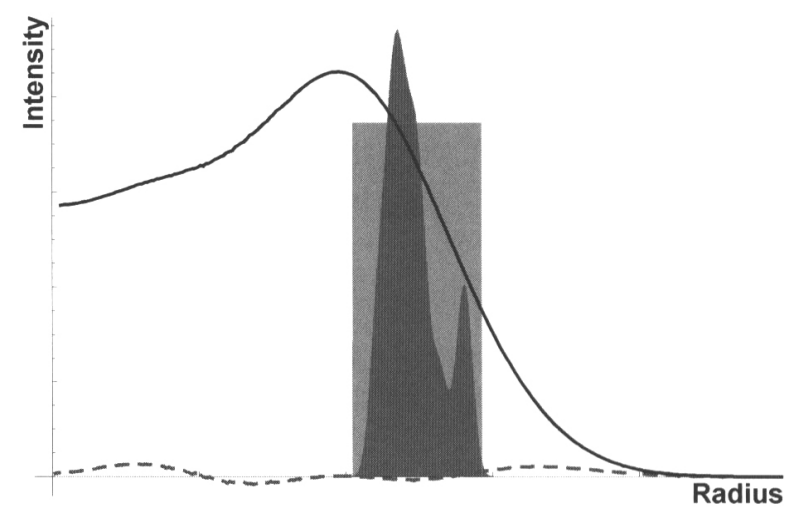

Fig. 3. Same as Fig. 2, but with realistic random noise and calibration errors. Notice the outward shift of the inner part of the reconstructed profile and the bias towards overestimating the emission in the inner region and underestimating it in the outer region.

the source center than expected for spherical shells of optically thin emission. Notice that, as said in Sect. 1, the Green Functions used in our GFD assume optically thin emission for the spherical shell. In order to further understand the implications of such central absorption (i.e. missing central emission at the source center with respect to what is expected for spherical shells of optically thin emission), and aware of the present limitation of our GFD method, we introduced central absorption in our synthesized noisy maps. Fig. 4 shows 


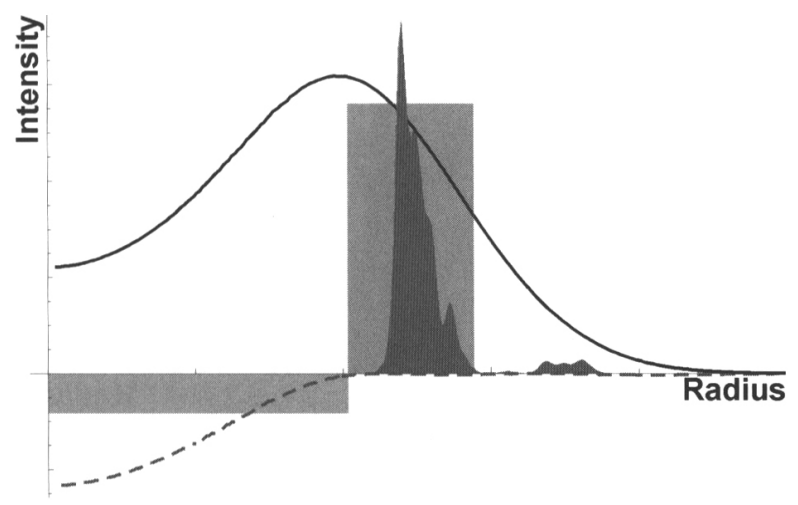

Fig. 4. Same as Fig. 3 but with a central absorber. The schematic of the central absorption indicates a complete blockage of the emission from the rear side of the shell for the extent of the drawing. In this case, the reconstructed profile is further biased towards larger radii and the reconstructed shell appears narrower than without the central absorber.

a schematic of the shell emission and the central absorption, the generated intensity profile and the deconvolved emission profile. The schematic of the central absorption in Fig. 4 indicates a complete blockage of the emission from the rear side of the shell for the extent of the drawing. The residuals are small everywhere, as before, but in the central region. These large central region residuals indicate the presence of the central absorption, as indeed we know is the case in this simulation. As it can be seen in Fig. 4, the recovered emission profile is not, under these conditions, a reliable representation of the shell profile. It bears resemblance to the shape of the profile recovered for the case without central absorption but the width of the profile is even narrower.

\section{GFD of Real Maps}

In Fig. 5 we use real data of SN 1993J from day 541. Without knowledge of the tests conducted and presented in the previous section one would not know how reliable this reconstruction is and how the true emission profile of the SN 1993J might be. However, the similarity of Figs. 4 and 5 is remarkable. From this similarity one is led to infer with caution that the emission profile of SN 1993J is probably that of a shell of width about $30 \%$ of size of the outer radius or somewhat less, since the deconvolved emission in Fig. 5 appears somewhat narrower than that in Fig. 4. Such a conclusion is in accord with previous experimental estimates but it does not help much to clarify further the situation. With respect to using the GFD method to determine the size of the radio supernova SN 1993J accurately, the prospects are not as good as expected but the application of the GFD method in SN 1993J reveals the 


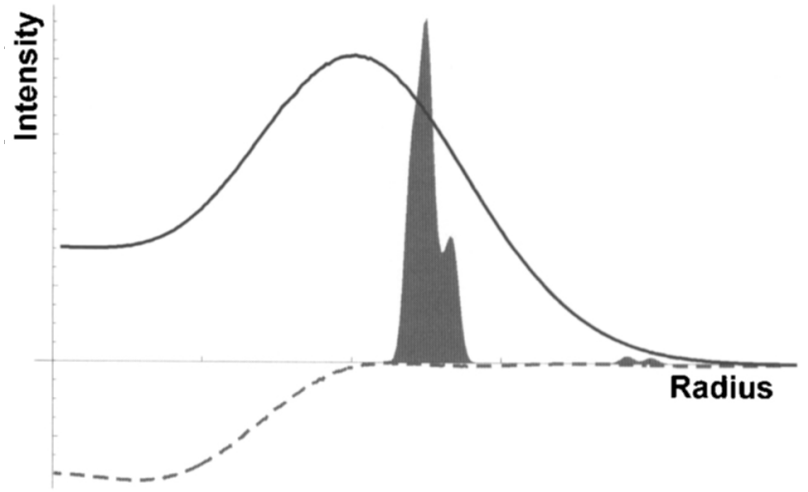

Fig. 5. Radial emission profile obtained from data of SN 1993J from day 541 after explosion. Notice its similarity to the profile reconstructed in Fig. 4.

presence of a strong absorption of emission at the source center with respect to what is expected for an optically thin spherically symmetric source.

\section{Other Radio Sources}

We have also applied the GFD to VLBI maps of $43.31+592$ in M 82 (maps courtesy of A. Pedlar) and we have obtained size estimates for $43.31+592$ very similar to those estimated with a different method [8, 9]. For 43.31+592 the GFD method seems to work better than for SN 1993J, perhaps because there is no central absorption. Another interesting case is SN $1987 \mathrm{~A}$ where the specific geometry is known. The analytic expression for $G_{\sigma}(r, \alpha)$ in this case would be a different one to that given in Section 1 since it corresponds to a planar and tilted emitter but, once determined, the GFD should work reasonably well unless absorption intervenes again.

\section{Expansion of SN 1993J}

Having found that the GFD method is not as well-suited as we had expected for determining the outer radius of SN 1993J accurately, we have developed other methods to determine the source size and shell width, abandoning hope of determining the profile of the emission within the shell. Of the new methods, which cannot be presented here, the best is one which we call the Common-Point Method [7] and which yields outer radius estimates accurate within $2 \%$ for cases when the shell size relative to the source size is not very different between observations (actually, changes in shell size map into changes in measured outer radius scaled down by a factor of 4). Combining these methods we have been able to produce a reliable expansion graph with data at $6 \mathrm{~cm}$ which is presented in Fig. 6 . 


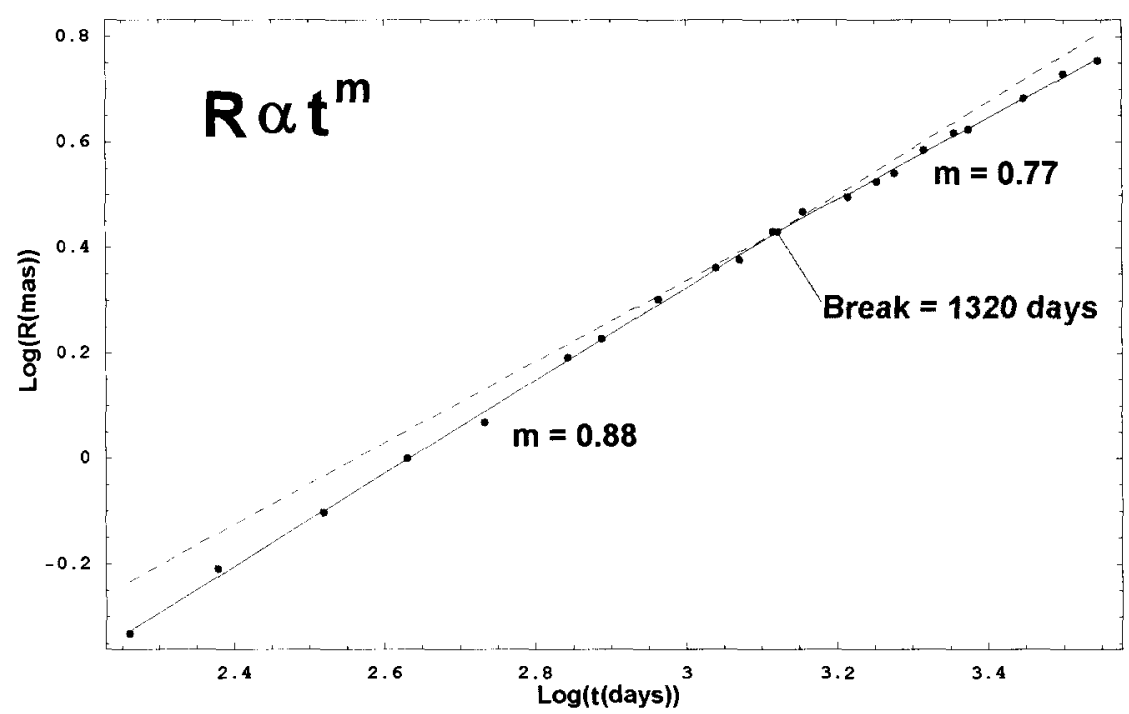

Fig. 6. Expansion of $\mathrm{SN}$ 1993J at $6 \mathrm{~cm}$, determined with the Common-Point Method [7]. $R \propto t^{m}$, where $R, t$, and $m$ are outer radius, age, and deceleration parameter, respectively.

\section{Conclusions}

A new method designed to determine the emission structure of a radio supernova works well for noiseless synthesized maps but does not work as well for noisy maps and for real maps. However, the method has been used to determine reliably the absorption in the central region of SN $1993 \mathrm{~J}$ with respect to emission expected from an optically thin spherical shell. This absorption manifests itself as large systematic residuals in the GFD procedure. This result, also reported by [4], is not due to any shape in the emission profile of the shell as pointed out earlier [3], but rather to absorption of the emitting sphere further away from us, the radiation of which is likely absorbed by the intervening ionized ejecta as pointed out by [5]. The GFD method, used with maps obtained from our observations and with simulated maps, gives support to emission from a shell, the width of which appears to be somewhat less than $30 \%$ of the outer radius of SN 1993J. The GFD method might work reasonably well in cases like SN 1987A whose geometry is known and the maps might soon have very high dynamic range.

Acknowledgement. JMM wishes to acknowledge support of this research from the Spanish Ministry of Science and Technology under contract AYA2002-00897. KWW wishes to thank the Office of Naval Research (ONR) for the 6.1 funding supporting this research. 


\section{References}

1. A. Alberdi and J.M. Marcaide: In: These proceedings

2. N. Bartel, et al. : Science 287, $112(2000)$

3. N. Bartel, M.F. Bietenholz, M.P. Rupen et al. : Astrophys. J. 581, 404 (2002)

4. M.F. Bietenholz, N. Bartel, M.P. Rupen: Astrophys. J. 368, 610 (2003)

5. C. Fransson and C.I. Björnsson: Astrophys. J. 509, 861 (1998)

6. J.M. Marcaide, A. Alberdi, E. Ros et al. : Astrophys. J. Lett. 486, L31 (1997)

7. J.M. Marcaide et al. : In preparation (2004)

8. A. Pedlar et al. : In preparation (2004)

9. A. Pedlar, T. Muxlow, and J. Riley: In: These proceedings

10. M.P. Rupen, A.J. Beasley, N. Bartel et al. : In: Radio Emission from Galactic and Extragalactic Compact Sources - IAU Coll. 164, eds. J.A. Zensus, G.B. Taylor, J.M. Wrobel (ASP Conf. Series 144, 1998) pp. 353-354 